\title{
EXPERIMENTAL CONSTRAINTS ON GLUINO MASSES AND SUPERSYMMETRIC THEORIES
}

\author{
G L KANE and J P LEVEILLE \\ Randall Laboratory of Physics, University of Michigan, Ann Arbor, MI 48109, USA
}

Received 11 January 1982

\begin{abstract}
We extend the analyses of $F$ dyet, and $F_{\text {ayet }}$ and $\Gamma$ arrar, of experimental scarches for gluinos, the supersymmetric partners of gluons Because of their large production cross sections, present data appears to exclude gluino masses below 3 5-6 $\mathrm{GeV} / \mathrm{c}^{2}$ and may be more restrictive Lifetime considerations give an upper limit on scaldr quark masses and on the scale of supersymmetry breakıng, and expenments sensitive to missing energy will provide interesting limits on the latter Since gluinos remain very light in many models, they will either be detected soon or many supersymmetric theorles will be excluded
\end{abstract}

1 Introduction Supersymmetric (SS) theones have been of considerable interest for some time, and have recently been studied intensively [1] because they incorporate scalars naturally into the theory and may dllow progress toward solving the gauge hierarchy problem

In SS theories conventional particles have associated particles differing by $1 / 2$ unit of spin For each quark and lepton there should exist a spınless partner, with the same mass Since these are not seen, SS must be broken If $S \subseteq$ is tc be of value for solving the gauge hierarchy problem, the scale of SS breakıng $\left(\Lambda_{\mathrm{SS}}\right)$ may [1] have to be of order $1 \mathrm{TeV}$ When SS is broken, the light scalars will get masses from rddiative corrections The masses are expected to be of order $m_{7}$, so it is not surprising that the scaldr partners of quarks and leptons have not yet been observed Broken global supersymmetries must [2] have a Goldstone fermion. the goldstino $(\mathrm{G})$ which couples to the gluon $(\mathrm{g})$ and its supersymmetric partner, the glumo ( $(\widetilde{\mathrm{g}})$

The gluinos have spin $1 / 2$ and we assume they carry the same quantum members of the gluons, $1 \mathrm{e}$ they are color octets Apparently it is hard to give much mass to gluinos, so it is rather surprising that they have not yet been seen Fayet, and Fdyet and Farrar, have already discussed this question $[1,3]$ In this paper we extend their andlyses, with several new results We drgue that existing data probably implies that gluno masses are larger than about $35-6 \mathrm{GeV}$, depending on $\Lambda_{\text {SS }}$ We suspect that existing data gives even stronger constraints, but normally data is not published in the form we need and will have to be reanalyzed by the experimenters involved Achieving a gluino mass ${ }^{+1}$ that large represents a senous challenge to SS theories that have a $\Lambda_{\mathrm{SS}}<1 \mathrm{TeV}$

It may be useful to point out where we go beyond the andlysis of Fayet, and Fayet and Farrar (1) For glunno masses beyond about $1 \mathrm{GeV}$ we can calculate production cross sections perturbatively Since gluinos are color octets these cross sections are quite large, up to twenty tumes those for quark parrs of simular mass, and give stringent limits It should be emphasized that our limits are conservative, since for heavy quarks the perturbative calculations are known to underestımate the cross sections (2) We consider larger gluno masses, and scales of SS breaking $\Lambda \lesssim 1 \mathrm{TeV}$, where the ggG vertex (1) dominates the gluino decay, (11) leads to

${ }^{+1}$ Given the couplings of a glumo, its constituent mass then fixcs its production cross section Since glumos are colored they wall be shiclded by strong color forces, most probably forming gluinoballs with gluons or quark -antiquark pairs These hadrons will have of order $1 \mathrm{GeV}$ of constituent mass even in the limit of massless gluinos Thus the limits on masses that go in the lagrangian should be reduced somewhat (by $<1 \mathrm{GeV}$ ) from the numbers we give Sunce it is not completely cledr how to do thdt, we will dways quote the mass that comes directly from analysis of experimental data and let the redder make the adjustment 
larger missing energy, and (w) leads to larger goldstino interaction cross sections $A$ ll these effects lead to better signatures hence easier detectability (3) We consider new production processes which give upper limits on gluino masses (or lower limits on $\Lambda_{\mathrm{SS}}$ ) in broken global SS theories (4) Since lifetimes of gluinos cannot be too long upper limits on $\Lambda_{\mathrm{SS}}$ or scalar quark masses can be obtaned (5) We emphasize the kinds of experiments needed to detect gluinos this is timely because present experuments could find them For example, in pp collisions at the ISR with $\mathcal{L}=10^{32} / \mathrm{cm}^{2}$ s) and running time of $10^{6} \mathrm{~s}$, if the gluno mass were $25 \mathrm{GeV}$ then $35 \times 10^{9}$ glumo parrs were produced, all with detectable signatures in principle

2 Glumo couplings Since gluinos are the partners of gluons they wll have the interactions shown in fig 1d The coupling at each vertex is the standard QCD coupling $g^{+2}$ In addition, for a broken SS a coupling to the goldstno is introduced as in fig $1 \mathrm{~b}$ Gauge invariance requires a magnetic type coupling, $h \overline{\mathrm{u}}_{\mathrm{G}} \sigma^{\mu \nu}$ $X \mathrm{u} \frac{a}{\mathrm{~g}} F_{\mu \nu}^{a}$ where $\mathrm{u}_{\mathrm{G}}$ and $\mathrm{u}_{\mathrm{g}}^{a}$ are spinors for a goldstino and gluino of color $a$, respectively, and $F_{\mu \nu}^{a}$ the gluon field strength The coupling strength $h$ is fixed by supercurrent algebrd ${ }^{\text {}} 3$ Indeed taking the matrix element of the supercurrent $S_{\mu}$ between a gluino and a gluon, including the goldstino pole term

$\$ 2$ The glumos are colored fermions and contribute to the $\beta$-function like quarks The numerical increase in $\alpha_{s}\left(Q^{2}\right)$ is small, however, and we may sately neglect it

${ }^{3}$ This is an extension to the colored states of the same argument given for photınos in ref [4] See also ref [5] and Dine et al, ief [1]

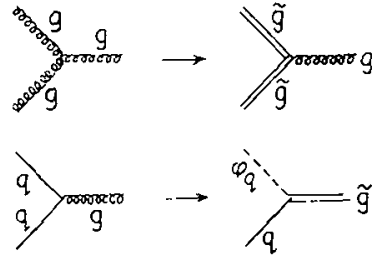

(a)

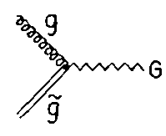

(b)
Fig 1 Gluino couplings in supersymmetric theories We represent gluons by g, gluınos by $\widetilde{\mathrm{g}}$, goldstınos by $\mathrm{G}$, quarks by $\mathrm{q}$, scalar partners of quarks by $\phi_{\mathrm{q}}$, and the photino by $\tilde{\gamma}$ the vertices of (d) will be prescnt in every supersymmetric theory when gluunos carry color The vertex of (b) is present in globdl superiymmetric theores and requiring zero divergence, yields $h=\tilde{m} / 2 \Lambda_{\mathrm{SS}}^{2}$ where $\widetilde{m}$ is the gluino current algebra mass and $\Lambda_{\mathrm{SS}}$ sets the scale of SS breaking, defined by $\left\langle 0\left|S_{\mu}\right| \mathrm{G}\right\rangle$ $=\Lambda_{\mathrm{SS}}^{2} \gamma_{\mu} \mathrm{u}_{\mathrm{G}}$

Some of our results only require the interactions of $\mathrm{ftg} \mathrm{la}$, for production of glumos va gluons They hold in any theory where the glumos are color octets In local SS the goldstino may become the helicity $1 / 2$ part of $d$ spin $3 / 2$ state so our results involving goldstinos may not directly hold However, since the gluinos will have to decay (see below), cssentrally equivalent results will be valid

3 Glutno lifettmes and interactions In a spontaneously broken global SS, the decay of the glumo proceeds dominantly via the vertex in fig $1 \mathrm{~b}$ We obtain the lifetume

$\tilde{\tau}_{G}=\left(033 \times 10^{-15} / h^{2} \Lambda_{\mathrm{SS}}^{4}\right)\left(\Lambda_{\mathrm{SS}} / M_{\mathrm{Z}}\right)^{4}(1 \mathrm{GeV} / \tilde{m})^{3} \mathrm{~s}$

Since current algebra arguments yield $h \approx \tilde{m} / 2 \Lambda_{\mathrm{SS}}^{2}$, this becomes

$\widetilde{\tau}_{\mathrm{G}} \approx 11 \times 10^{-15}\left(\Lambda_{\mathrm{SS}} / M_{\mathrm{Z}}\right)^{4}(1 \mathrm{GeV} / \tilde{m})^{5} \mathrm{~s}$

If observations imply $\tilde{m}>3 \mathrm{GeV}$, and $\Lambda_{\mathrm{SS}}<1 \mathrm{TeV}$ for the cases of interest, then $\tilde{\tau}<07 \times 10^{-13}$ s If $\widetilde{\mathrm{g}}$ is produced with $\gamma=20$, it will travel typically 04 $\mathrm{mm}$ On the other hand, if $\widetilde{m} \simeq 1 \mathrm{GeV}$, and $\Lambda_{\mathrm{SS}}=1$ $\mathrm{TeV}$, it goes $^{6}$ times further, typically $030 \mathrm{~m}$ with, of course, some going over a meter Note that eq (2) provides an interesting upper limit on $\Lambda_{\mathrm{SS}}$ for a given $\widetilde{m}$ (see fig 2) If data excludes production of a gluino which travels more than about $10 \mathrm{~cm}$ (see below), then any theory must sdtisfy $\Lambda_{\mathrm{SS}} / \widetilde{m}^{1} 5 \leq 1000$, with $\Lambda_{\mathrm{SS}}$ and $\widetilde{m}$ in $\mathrm{GeV}$ units

If the $\widetilde{g g G}$ vertex is suppressed or absent as perhaps could occur in a local SS, the glunno wll decay via a virtual scalar quark to a quark-antıquark pair and a photino $(\tilde{\gamma})$ (provided that the gluino is heavier than the photino)

For the mode $\widetilde{\mathrm{g}} \rightarrow \mathrm{q} \overline{\mathrm{q}} \tilde{\gamma}$, the lifetume is $\tilde{\tau}_{\widetilde{\gamma}}=08 \times 10^{6}\left(m_{\mu} / \widetilde{m}\right)^{5}\left(M_{\phi} / M_{\mathrm{W}}\right)^{4} \mathrm{~s}$

$M_{\phi}$ is the lightest scalar quark mass associated with quark s lighter than the gluino By comparison, the $\widetilde{\gamma}$ mode dominates if $M_{\phi}<009 \Lambda_{\mathrm{SS}}$, if $M_{\phi}=M_{\mathrm{W}} / 2$, the photino mode dominates for $\Lambda_{\mathrm{SS}} \gtrsim 400 \mathrm{GeV}$ (see fig 2) 


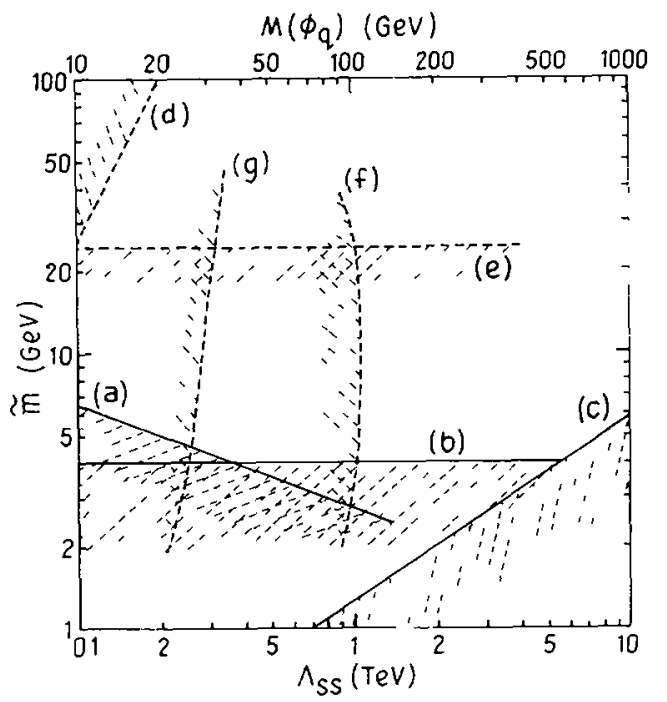

Tig 2 Fxcluded region in the $\tilde{m}-\Lambda$ SS plane Soldd curves are

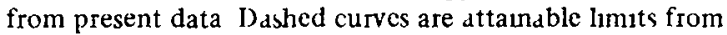
future cxperiments (d) Valıd if $\tilde{\mathrm{ggG}}$ vertex is present (b) I rom $\widetilde{\mathrm{g}} \rightarrow \mathrm{q} \overline{\mathrm{q}} \tilde{\gamma}$ with subsequent $\tilde{\gamma}$ interaction in a beam dump detector I his curve is always present We have assumed $M_{\varphi_{\mathrm{q}}}$ $=M_{\mathrm{W}} / 2$ (c) Gives upper limit on $\Lambda_{\mathrm{SS}}$ (lower scale) or $M_{\phi_{\mathrm{q}}}$ (upper scale) from the requirement that $\widetilde{g}$ lifetime not be too long (d) Upper limit on $\tilde{m}$ from double goldstino production at Isabelle (e) The region below this line would be excluded by a fallure to detect gluino production by an SPS detector with $\mathcal{L}=10^{29} / \mathrm{cm}^{2}$ (f) The region to the left is excluded if 100 events of $\mathrm{G}+\widetilde{\mathrm{g}}$ production are not detected at Isabelle (g) Same as (f) tor FNAL collider

Thus we expect that experiments sensitive to neutral hadrons that can travel centumeters or meters will give a lower limit on the gluino mass When a gluino is produced it will be shielded to make a color singlet hadron Most probably the gluıno will bind with a gluon, because of the octet binding forces, though sometımes the glumo could attach to d color octet $\mathrm{q} \overline{\mathrm{q}}$ pair The electrncally neutral, color singlet, hadron will interact like a normal hadron, with a total interaction probablity like that of a kaon or a $\mathrm{D}^{0}$, with $\sigma_{\text {tot }} \sim$ few $\mathrm{mb}$ As observed $[1,3]$ by Fayet and Farrar, and as we will reaffirm below, any objects produced with several $\mu \mathrm{b}$ cross sections, and having such lifetımes and interactions, would probably have been observed

4 Gluino and goldstino production Once the small mass range $\widetilde{m}<1 \mathrm{GeV}$ is excluded by the absence of long-lived, electrically neutral, strongly interacting hadrons, we can reliably use perturbatıve QCD to cal-<smiles>CC(=O)C(C)C</smiles>

(a)

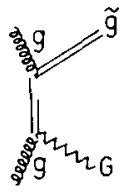

(b)

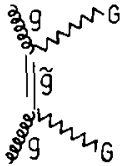

(c)
Tig 3 Production mechanism for gluinos and goldstinos (a) $1 s$ present in any theory where gluinos carry color It gives the cross sections of fig 4 for color octet gluinos (b) and (c) are present in globally supersymmetric theories and give upper limits of $\mathrm{f}_{1 \mathrm{~g}} 2$

culate (lower limits on) the production cross sections, and these are very large for color octet gluinos Further, in any theory where there is a ggG coupling, the double or single durect goldstino production cross (figs $3 \mathrm{~b}, 3 \mathrm{c}$ ) sections increase with $\widetilde{m}$ and the absence of experumental detection of such events will give an upper limit on $\tilde{m}$

We show the gluıno parr-production mechanisms for $\mathrm{pp}$ and $\mathrm{p} \overline{\mathrm{p}}$ collisions in fig $3 \mathrm{Fig} 4$ shows the production cross section versus the gluino mass $\tilde{m}$ for a number of beam energies Since the curves fall rapidly, a small error in estimating the cross section

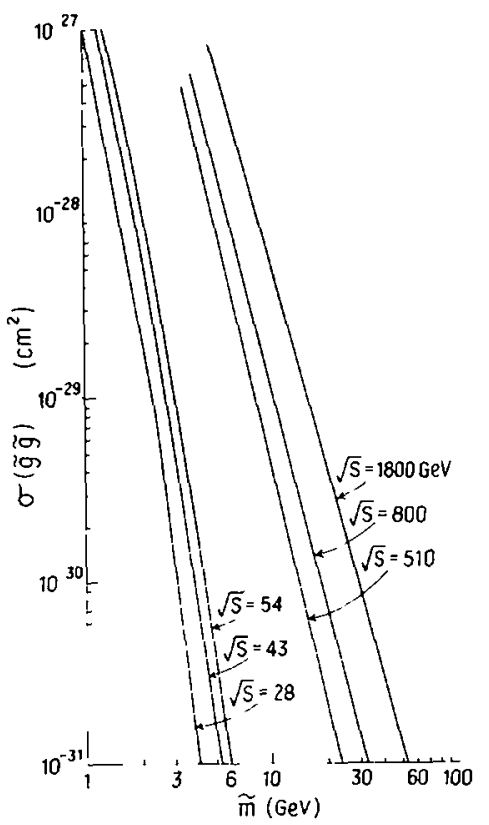

Fig 4 Gluino production cross sections computed from fig 4a, including scaling violdtions, as parametrized by Bdier et al in ref $[11]$ for severdl values of $\sqrt{s}$ (in GcV) 
limit has little effect on the dssociated mass limit Once again these are expected to be conservative lower limits since production of $c \bar{c}$ and $b \bar{b}$ is larger than the perturbative prediction

It should be emphasized that the cross sections are quite large The actual calculation includes not only the diagram of fig $3 a$, but the crossed graph, the direct gluon pole term, and the production via quarks, $q \bar{q}$ $\rightarrow \mathrm{g} \rightarrow \overline{\mathrm{g}} \overline{\mathrm{g}}$ In the region of interest the subprocess shown is the largest one in the Feynman gauge, and to understand the size of the cross section we can compdre it to q $\bar{q}$ production With generators $F^{a}$ in the octet representation and $\lambda^{a / 2}$ in the fundamental representation, we have for equal kinematics, and infinite energy,

$\frac{\sigma(\mathrm{gg} \rightarrow \tilde{\mathrm{g}} \tilde{\mathrm{g}})}{\sigma(\mathrm{gg} \rightarrow \mathrm{q} \overline{\mathrm{q}})} \approx \frac{\operatorname{tr} F^{a} F^{a} F^{b} F^{b}}{\operatorname{tr} \lambda^{a} \lambda^{a} \lambda^{b} \lambda^{b} / 16}=135$

For the actual calculation ${ }^{\neq 4}$ the gluno pair cross section varies from 16-20 tumes the cross section for production of a parr of quarks of the same mass

\section{Rectroactive analysts of data It is obviously dif-} ficult to analyze existing expenments to see what limits they put on gluino masses It hds even been suggested that experımenters only find what they are looking for We will abstract from past data some estımates on what might have been seen, our results are summarized in fig 2 We want to emphasize that they are only estimates, and should not be taken as firm limits until experimenters have analyzed theır own data with a full knowledge of backgrounds, cuts, etc Experiments in progress can set stgnificantly better limits than we obtain if they are analyzed with glumo (or goldstino) detection in mind, and experiments at SPS, ISABELLE or FNAL can go to very high masses

(a) Small glutno masses and longer lifetumes As discussed above, if $\widetilde{m}$ is of the order of $1 \mathrm{GeV}$ the lifetime is farrly long Fayet and Farrar have already argued that this is not allowed by data, and we agree The case can be made very strong For small $\tilde{m}$, while pertur-

₹4 In all calculations we have assumed that the glumos are Dirdc fermions If they dre Majorana fermions there is an extra factor of $1 / 2$ in the phase space Since the cross sectrons are falling cxponentially with the masses of the produced gluinos this docs not affect our final conclusions substantidlly bdtive calculations are not reliable, the production cross section will not be smaller than that of fig 4 , so $\sigma \gtrsim 1 \mathrm{mb}$ Produced gluinos will be shielded by gluons or $\mathrm{q} \overline{\mathrm{q}}$ octet pairs, so an electrically neutral hadron will be produced, travel a distance from mullimeters to meters, and decay into an even number of charged hadrons (often four or more hadrons) which do not point back to the production vertex There is missing energy because of the goldstino (or photino) but no charged leptons The shelded gluno will interact with a total cross section in the mb range The experıment [6] of Gustafson et al can put limits of order $1032 \mathrm{~cm}^{2}$ on any neutral object produced in appropriate regions of $p_{\mathrm{T}}$ and $X_{\Gamma}$ whuch goes several meters and then interacts with a millibarn cross section Experiments in hyperon beams may [7] be able to put limits of order $10^{-3}-10^{-4}$ tumes the $\Lambda$ cross section on objects which go d few meters and decay into an even number of charged prongs which do not point back to the production vertex In hydrogen bubble chambers there are strong restrictions ${ }^{+5}$ on events which would give a visible gap and an even number of prongs (neutrons give a recol proton and an odd number of prongs) Altogether, we think it is convincing that objects with the properties of light gluinos are not produced with cross sections of even a few $\mu$ b, so $\widetilde{m}$

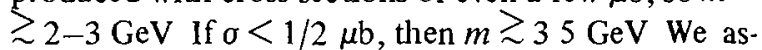
sume fixed target pp collisions with $\sqrt{s} \simeq 28 \mathrm{GeV}$ for these numbers, they vary a little for other energies or beams

(b) Beam dump experiments Once the mass is as large as established in (a) above, most gluınos decay withun a few $\mathrm{cm}$, and either beam dump or missing energy detectors will be most restrictive In beam dump experiments the goldstıno will interact in the detector, giving no charged lepton and thus candidate neutralcurrent (NC) events Recent expenments looking for axions quote [8] an upper limit $(2 \sigma) \sigma_{1 n t} \leqslant 2 \times 10^{-67}$ $\mathrm{cm}^{4}$ where $\sigma$ is the production cross section for the gluino in our case and $\sigma_{\text {int }}$ the interaction cross section for the goldstino Assuming that the goldstino interdction is like a charged-current neutrino interaction, and an average energy of $60 \mathrm{GeV}$ for the goldstino (a typical $\nu$ energy in such an experiment), we find again that $\sigma \lesssim 1 / 2 \mu$ b or equivalently $\widetilde{m} \gtrsim 35$ $\mathrm{GeV}$

\$5 We thank B Roe and $\mathbf{R}$ Rau for discussions on this data 
For some ranges of $\widetilde{m}$ and $\Lambda_{\mathrm{SS}}$ this result can be considerably strengthened by further data analysis First, in any theory with a ggG vertex the goldstino interaction wil be much larger than the $\nu$ charged cross section Indeed the goldstino can interact with protons in the detector by fusing with a constituent gluon, which yields the rate

$\sigma_{\mathrm{G}}=\left(\pi / \Lambda_{\mathrm{SS}}^{2}\right)\left(\tilde{m} / \Lambda_{\mathrm{SS}}\right)^{2} x G\left(x, \widetilde{m}^{2}\right)$, where $x \equiv \widetilde{m}^{2} / s$ and $x G\left(x, \widetilde{m}^{2}\right)$ is the gluon distribution function evaluated at $x$ and $Q^{2} \approx \widetilde{m}^{2}$ For the range of masses and energy considered we may safely use $x G \simeq 3(1-x)^{5}$ which yields

$\sigma_{\mathrm{G}}=\left(37 / \Lambda_{\mathrm{SS}}^{4}\right) \mathrm{s} x(1-x)^{5} \mathrm{mb}$

with $s$ and $\Lambda_{\mathrm{SS}}$ in GeV units Using $60 \mathrm{GeV}$ for a mean goldstino energy and $\Lambda_{\mathrm{SS}}=300 \mathrm{GeV}$, we find that $\sigma_{G}>\sigma_{\nu}$ in the range $1 \mathrm{GeV} \lesssim \widetilde{m} \lesssim 6 \mathrm{GeV}$ and the goldstino interaction cross section is increased by a factor of 4-6 over the contributions considered previously This strengthens the previous limits and pushes $\tilde{m}$ to about $45 \mathrm{GeV}$ Second ${ }^{\text {t }}$, a $\nu$ NC event has large missing $p_{\mathrm{T}}$ for the hadrons, and a spectrum of visible hadron energy $\left(E_{\mathrm{vK}}\right)$ which peaks at low $E_{\mathrm{vis}}$ and does not have a long tall A goldstino induced event, on the other hand, wlll have considerably larger $E_{\mathrm{v} 1 \mathrm{~s}}$ (thus it could not dccount for any extrd events at small $E_{\text {ns }}$ ) and much smaller $\left(p_{1} / p_{\mathrm{L}}\right)_{\text {had }}$ Cuts on these varudbles could eliminate most $\nu$ NC candidates and allow a small goldstino signal to be found, or give a limit well below $1 \mu \mathrm{b}$

The photino interaction cross section is dominated by the process $\gamma+q \rightarrow q+g$, with a scalar quark exchanged, as discussed by Fayet [3] This gives a cross section

$$
\begin{aligned}
& \sigma_{\mathrm{tnt}}=12 \times 10^{-38} E\left(M_{\mathrm{W}} / M_{\phi}\right)^{4} \\
& \times \sum_{q} \int_{\widetilde{m}^{2} / s}^{1} x q(x)\left(1-m^{2} / x s\right)^{3} \mathrm{~d} x e_{\mathrm{q}}^{2}\left(\mathrm{~cm}^{2}\right)
\end{aligned}
$$

with $E$ in $\mathrm{GeV}$ We assume that the lightest scalar quark has $M_{\phi}=M_{\mathrm{W}} / 2$ Then combining this with the beam dump limit gives curve (b) of fig 2, drawn for fixed $M_{\phi}$ and $\Lambda_{\mathrm{SS}}$ (in a particular theory, $M_{\phi}$ may depend on $\Lambda_{\mathrm{SS}}$ ) Even if the goldstino is not present

\footnotetext{
\#6 We thank J Morfin for discussions on this analysis
}

the photino decay together with the beam dump data already provides a stringent lower limit on $\widetilde{m}$

(c) Missing energy and $p_{T}$ experiments The most powerful limits will come from experiments, at Tevatron and collider energes, which constran missing energy and momcntum as well as possible Agan, we emphasize SS theories with a ggG vertex, but our remarks dpply also to theones without such a vertex so fdr as a lower limt on $\tilde{m}$ is concerned The upper limit on $\widetilde{m}$ depends crucially on such a vertex

Consider an experiment at the ISR pp collider with a typical integrated luminosity of $10^{37} / \mathrm{cm}^{2}$ Then if $\sigma>10^{-33} \mathrm{~cm}^{2}$ it had $10^{4}$ gluino pairs produced This corresponds to $\widetilde{m} \geq 10 \mathrm{GeV}$ if gluinos were not found Similarly, consider $\int \mathcal{L} \mathrm{d} t=10^{35} / \mathrm{cm}^{2}$ at the SPS collider Then $10^{4}$ events correspond to $\sigma=10^{-31}$ $\mathrm{cm}^{2}$, or $m \geq 24 \mathrm{GeV}^{\prime}$

Could such events have been seen already? The1r signature is farly dramatic The glunnos are produced in the central region, and decay via $\tilde{\mathrm{g}} \rightarrow \mathrm{gG}$ The gluon gives a hadronic jet so there is a parr of acoplanar jets, plus a lot of missing transverse cnergy and momentum, and no prompt charged leptons Typically, about $25 \%$ of the energy will go into the central collision, so $1015 \%$ of the total energy and about half of the central energy will be mussing Certainly $10^{4}$ such events could be found in ISR or SPS experiments specifically lookung for them in the near future

6 Upper limits Since the cross sections for double goldstıno production grow as $\widetilde{m}$, they give upper limits on $\tilde{m}$ or lower limits on $\Lambda_{\mathrm{SS}}$ if a signal is not found The signature for goldstino pair production is an event with an interaction and beam jets but essentially no central region energy The goldstıno-gluino production is edsier to see as $\widetilde{\mathrm{g}} \rightarrow \mathrm{gG}$ giving one jet (or $\widetilde{\mathrm{g}}$ $\rightarrow q \bar{q} \tilde{\gamma})$, with no partucles detected in the opposite direction Neither type of event has prompt charged leptons These give the future curves $d, f, g$ of fig 2

7 Gluino masses Fayet has discussed [3] gluno in some detall In the unbroken theory $\widetilde{m}=0$ since they dre degenerate with gluons Gluinos will not get mass at tree level even if SS is broken since color symmetry is unbroken of course, an explicit ad hoc mass term could be written for gluinos, but that explains nothing Fayet [9] suggests one mechanısm to give gluinos a Dirac mass of order $1 \mathrm{GcV}$, but it requires 
adding a color octet of "paragluunos" and may not be tolerable if grand unification is a goal [10] SS theones often have U(1) symmetries (called R-invariances [9]) which must be broken to allow a Majorana mass for $\tilde{\mathrm{g}}$

So far, not much attention has been pard to gluino masses when constructung models Perhaps by focusing attention on models where $\widetilde{m}$ satisfies the constraints of fig 2, progress can be made towards finding a realistic theory

8 Conclusions Since glumos tend to be lighter than other supersymmetric partners, and are produced with large cross sections, they should be considered as the prime hope in deciding experimentally whether nature is supersymmetnc We think, conservatively, that gluinos would probably have been detected if their masses were in the excluded region of fig 2 , basically, $\widetilde{m} \gtrsim 4 \mathrm{GeV}$ Analysis of existıng datd by expernmenters, and experıments in progress, can strengthen these limits considerably if glumos are not detected Since glumo properties depend on the scale of SS breakıng and on scalar quark masses, interesting upper/ lower limits on all of these are implied by upper limits on lifetumes of long-lived neutral hadrons and on production cross sections

We appreciate instructive conversations about experimental constraints with a number of colleagues, partıcularly B Roe, C Baltay, R Rau, M Longo, $\mathbf{J}$ Morfin and $O$ Overseth We have benefited from theoretical discussions with S Raby, B deWit, D R T Jones, and particularly M B Einhorn and $S$ Dimopoulos This work was supported in part by the US Department of Energy

\section{References}

[1] Recent references related to the questions we discuss include $P$ Fayet, Supersymmetry, particle physics, and gravitation, in Unification of the fundamental particle interactions, eds S Ferrara, J Fllis and $P$ van Nieuwenhuizen (1980), G R Farrar, Supersymmetry in nature, Lectures at the Intern School of Subnuclear Physics (Ence, 1978), M Dine, W Tischler and M Srednick1, Nucl Phys B189 (1981) 575,

N Sakd1, lohuku Unıv prepnnt \#TU/81/225, L $\Gamma$ Ibancz and G G Ross Oxford Univ preprint (1981), F Witten, Prunceton preprint (1981), S Dimopoulos and S Raby, Nucl Phys B 192 (1981) 353 , S Dimopoulos and H Georg1, Nucl Phys B193 (1981) 150 , G Barbiellini ct al, LCP Study, DeSY 79/67 (1979)

[2] P Fayet and J Illopoulos, Phys Lett 51B (1974) 461

[3] G R Farrar and P Fayet, Phys Lett 198 (1978) 442 , 76B (1978) 575 , $P$ F ayet, Phys Lett 78B (1978) 417

[4] N Cabibbo, G R Farrar and L Maranı Phys Lett 105B (1981) 155

[5] P Fayet, Phys Lett 70B (1977) 461

[6] H R Gustafson, C A Ayre, L W Jones, M J Longo and P V R Murthy, Phys Rev Lett 37 (1976) 474

[7] O Overseth, private communication

[8] See e g, Proc XXth Intern Conf on High energy physics, Madison, eds , L Durand and L G Pondrom (1980)

19] P Fayet, Nucl Phys B90 (1975) 104, A Salam and J Strathdec, Nucl Phys B87 (1975) 85

[10] S Dimopoulos, private communication

[11] R Bdier et al, Z Phys C2 (1979) 265 